\title{
Changes in the Protein Profile of Streptomyces griseus during a Cycloheximide Fermentation
}

\author{
KEVIN H. DYKSTRA AND HENRY Y. WANG \\ Department of Chemical Engineering \\ University of Michigan \\ Ann Arbor, Michigan 48109
}

\section{INTRODUCTION}

The production of the antifungal agent cycloheximide by Streptomyces griseus is typical of many antibiotic fermentations. In the initial stage of the fermentation, cell growth is extensive, while little antibiotic is produced. After roughly 24 hours, the biomass concentration remains relatively constant and accumulation of product in the fermentation broth begins. It has been shown that production of cycloheximide is feedback regulated and that extracellular cycloheximide is degraded.'

One approach to alleviating the problems of feedback regulation and product degradation is to continuously remove the product from the fermentation broth. In the case of cycloheximide, both the rate of production and the total amount of cycloheximide produced are dramatically increased when the antibiotic is adsorbed from the fermentation broth using neutral polymeric resin (FIGURE 1). ${ }^{2}$

In order to gain insight into the types of metabolic changes that occur with continuous product extraction, this study will focus on elucidating the mechanism of feedback regulation in this system. In the first part of this work, a computer model of the fermentation will be used to examine the effects of different modes of feedback regulation. In the second portion, Two-Dimensional Polyacrylamide Gel Electrophoresis will be used to directly examine the amount and type of cellular proteins as a function of time.

\section{THEORY: FERMENTATION MODELING}

Feedback regulation can occur at two different levels. In feedback inhibition, a regulatory substance interacts directly with a synthesizing enzyme to decrease its activity. Feedback repression is the result of the interaction of a regulatory substance with the gene coding for a particular enzyme, thereby decreasing the synthesis of that enzyme. Thus, feedback inhibition results in a decrease in the specific activity of an enzyme, whereas feedback repression results in a smaller amount of enzyme. To examine the effects of these different modes of feedback regulation, a computer model of the fermentation was constructed.

It was desired to account for cell growth, substrate consumption, and cycloheximide accumulation. Because the fermentation medium is complex, with a large amount of undissolved solids, there are few data available on the cell growth profile. 
However, it has been observed that the dry cell weight increases from roughly $1 \mathrm{~g} / \mathrm{L}$ to $10 \mathrm{~g} / \mathrm{L}$ in approximately 24 hours and that the cell mass concentration changes little after that. The choice of a model for cell growth was therefore somewhat arbitrary. A logistic growth model, ${ }^{3}$ given by

$$
\frac{d X}{d t}=\mu_{\max }\left(1-\frac{X}{X_{\max }}\right) X,
$$

where $X$ is the biomass concentration $(\mathrm{g} / \mathrm{L}), \mu_{\max }$ is the maximum specific growth rate, and $X_{\max }$ is the maximum biomass concentration $(10 \mathrm{~g} / \mathrm{L}),{ }^{4}$ was chosen. The maximum specific growth rate was found to be $0.3 \mathrm{~h}^{-1}$.

In this model, consumption of substrate was assumed to be related to cell growth,

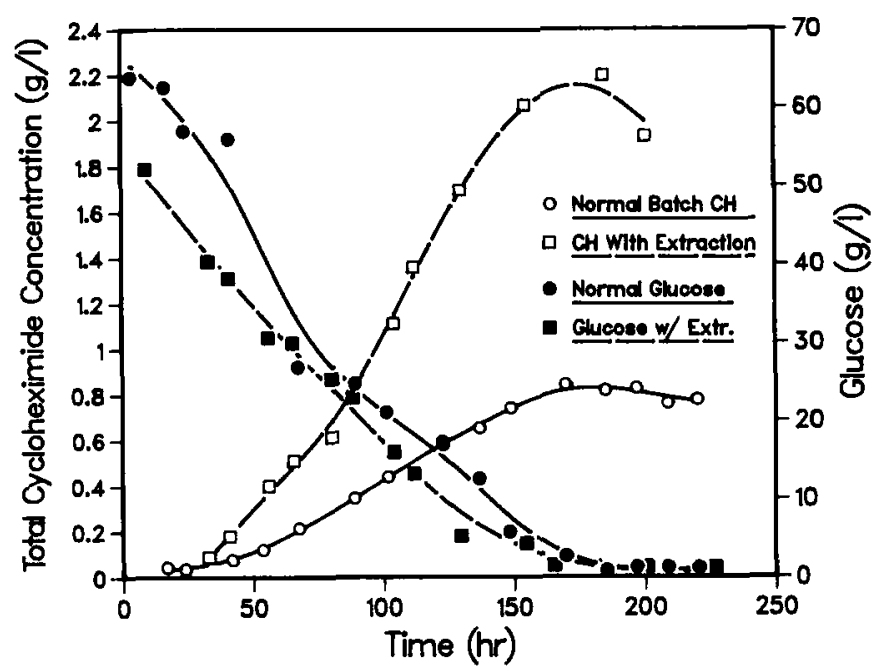

FIGURE 1. Typical fermentation profiles showing the effect of continuous product extraction on cycloheximide production.

cycloheximide synthesis, and cell maintenance. The differential equation describing substrate utilization is thus

$$
\frac{d S}{d t}=-\left(Y_{x / s}\right)^{-1} \frac{d X}{d t}-\left(Y_{p / s}\right)^{-1} R_{s}-m X
$$

where $Y_{x / s}$ and $Y_{p / s}$ are the yield coefficients for cell mass (g cells/g glucose) and cycloheximide production ( $\mathrm{g} \mathrm{CH} / \mathrm{g}$ glucose), respectively; $R_{3}$ is the rate of cycloheximide synthesis ( $\mathrm{g} \mathrm{CH} / \mathrm{L} \cdot \mathrm{h}$ ); and $m$ is the specific maintenance coefficient (g glucose/g cell.h).

The specific maintenance utilization has been determined experimentally for this system by measuring the substrate consumption of idiophase cells subjected to varying degrees of cycloheximide feedback regulation. ${ }^{5}$ With the cells in this state, the 
substrate consumption due to metabolic processes independent of cycloheximide synthesis (i.e., cell maintenance) could be extrapolated. The maintenance coefficient, $m$, was found to be $\sim 0.03 \mathrm{~g}$ glucose $/ \mathrm{g}$ cell $\cdot \mathrm{h}$.

During the growth phase, cycloheximide production is negligible, so glucose utilization can be related to cell growth and maintenance alone. With a knowledge of the maintenance requirement, $Y_{x / s}$ could be found by fitting the glucose utilization profile early in the fermentation. Using this approach, $Y_{x / s}$ was found to be around 3.0 $\mathrm{g}$ cells/g glucose. Experimental evidence in this lab and from Kominek ${ }^{1}$ indicates that the glucose biomass yield during the growth phase is much higher than that which is theoretically possible if glucose were the only carbon source. Therefore, the unusually high yield can likely be ascribed to the complex fermentation medium, which contains a high concentration of amino acids and other components that can be used as alternative carbon sources for cell growth. Later in the fermentation, glucose appears to be preferentially utilized, and it has been shown to be required for antibiotic production. ${ }^{1,5}$

The yield of cycloheximide on glucose was found in a manner similar to that used for the biomass yield. During the production phase, glucose consumption can be attributed to cycloheximide production and cell maintenance alone. Although several different models for cycloheximide production were tested in this work, a value for $Y_{p / s}$ of $0.13 \mathrm{~g} \mathrm{CH} / \mathrm{g}$ glucose resulted in very good agreement with the experimental glucose profile.

In modeling the production of cycloheximide, there are three characteristic fermentation profiles that the model must describe:

(1) In a batch fermentation, synthesis begins after roughly 24 hours and continues until glucose in the medium is exhausted. ${ }^{1.5}$

(2) When glucose is fed to the fermentation, antibiotic accumulation continues until the extracellular concentration reaches roughly $1000 \mathrm{mg} / \mathrm{L}_{;}^{1,5}$ then it stops due to feedback regulation.

(3) When the product is removed continuously by resin addition, the rate of accumulation and the overall production are dramatically increased. ${ }^{2}$

The net accumulation of antibiotic was taken as the difference between the rates of synthesis and degradation:

$$
\frac{d P}{d t}=R_{\mathrm{s}}-R_{\mathrm{d}}
$$

For this system, product degradation has been shown to be adequately modeled as a first-order process with respect to cycloheximide concentration: ${ }^{5}$

$$
R_{\mathrm{d}}=K_{\mathrm{d}} P .
$$

The degradation rate constant has been found to depend on both the $\mathrm{pH}$ of the medium and the biomass concentration. ${ }^{5}$ However, under normal fermentation conditions, these variables remain constant and $K_{\mathrm{d}}$ could be taken as constant at an experimentally determined value of $5 \times 10^{-3} \mathrm{~h}^{-1}$.

Because the work presented thus far in the literature ${ }^{1}$ has suggested that cycloheximide production is feedback inhibited, a model was initially tested in which a rate-limiting enzyme was present at constant level and was subject to noncompetitive 
end-product inhibition. Following an initial lag phase of roughly $24 \mathrm{~h}$, the rate of cycloheximide synthesis was given by

$$
R_{\mathrm{s}}=Q_{p m} X\left(1+P / K_{l}\right)^{-1} \text {. }
$$

The maximum specific synthesis rate, $Q_{p m}$, was found by dividing the initial total (broth plus resin) accumulation tate by the biomass concentration $\left(X_{\max }\right)$ for a fermentation with continuous product removal $\left(Q_{p m}=1.5 \times 10^{-3} \mathrm{~g} \mathrm{CH} / \mathrm{g}\right.$ cells $\left.\cdot \mathrm{h}\right)$. This value represents the highest experimentally observed initial cycloheximide production rate. The value for the inhibition constant, $K_{l}$, was found by observing which gave the best fit with the experimental cycloheximide profile. This system of ordinary, nonlinear first-order differential equations was solved using a fourth-order Runge-Kutta algorithm, and the quality of fit was evaluated by examining the sum of the squared errors between experimental data points and the simulation.

Because of the paucity of data on the cell growth kinetics for this system, the simple growth model presented above did an adequate job of describing cell growth. A comparison of the experimental glucose consumption profile with the simulated profile for a typical batch fermentation (FIGURE 2) shows close agreement between the simulation and the experimental data. Although models for cycloheximide synthesis simulating several different modes of feedback regulation were tested (see below), the fit for the glucose profile remained very good.

A comparison between the simulated cycloheximide concentration profile for the constitutive, inhibition model and the experimental batch profile is also shown in Figure 2. It can be seen that the agreement between the simulation and the experiment is not especially close. Early in the production phase (24-70 h), the simulated rate of cycloheximide accumulation is much higher than that seen experi-

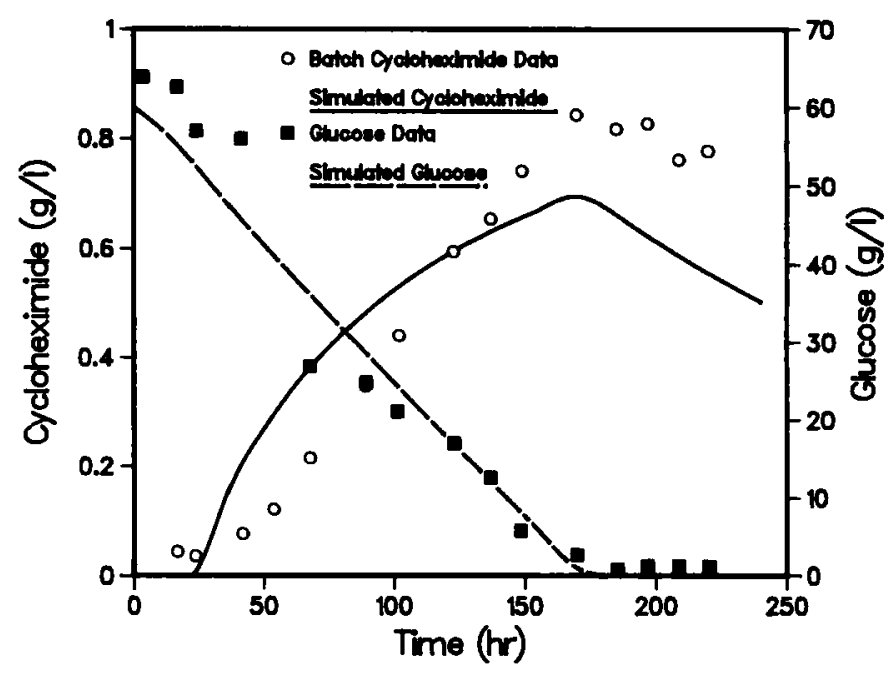

FIGURE 2. Simulated fermentation profiles using constitutively produced enzyme and feedback inhibition. Note that the simulated glucose profile agrees well with the experimental data, while the cycloheximide profile does not. 


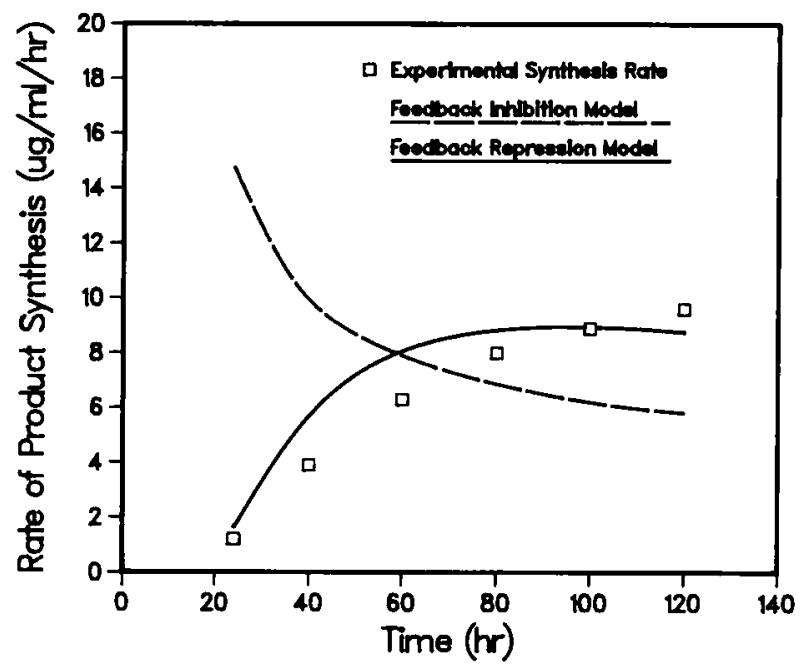

FIGURE 3. Comparison of simulated versus experimental cycloheximide synthesis rate profiles. The inhibition-only model is in fundamental disagreement with the experiment.

mentally. Later, the simulated accumulation rate decreases as the effects of endproduct degradation and inhibition become important. During this part of the fermentation, however, the experimental rate of cycloheximide accumulation has increased substantially.

To more closely evaluate this effect, equation 3 was rearranged to give

$$
R_{\mathrm{s}}=\frac{d P}{d t}+K_{\mathrm{d}} P
$$

The experimental rate of cycloheximide accumulation was found by graphically differentiating a smooth curve drawn through the experimental cycloheximide profile. Then, using the degradation rate and cycloheximide concentration, equation 6 could be used to find the experimental rate of cycloheximide synthesis.

In FIGURE 3, it can be seen that the experimental rate of synthesis is low in the early stages of the fermentation. Following initiation of the production phase at around $24 \mathrm{~h}$, the synthesis rate can be seen to increase monotonically over the course of the fermentation. In the fed-batch fermentation (data not shown), the synthesis rate goes through a maximum before reaching a final, steady-state value equal to the rate of product degradation. However, the simulated rate of synthesis for the constitutive model starts out very high and then decreases monotonically throughout the production phase. Thus, there is a fundamental discrepancy between the experimental data and the inhibition-only model.

It was because of these problems that a model for cycloheximide synthesis that included the effect of varying synthetic enzyme concentration was tested. It was necessary to introduce a new state variable, $E$, which represents the intracellular concentration of an uncharacterized rate-limiting enzyme in $\mathbf{g}$ protein $/ \mathbf{g}$ cells. $\mathbf{A}$ 
similar model has been used to describe the concentration of anhydrotetracycline oxygenase in the fermentation of Streptomyces aurofaciens for the production of tetracycline. ${ }^{6}$ The rate of cycloheximide synthesis in equation 3 is now proportional to the intracellular enzyme concentration:

$$
R_{\mathbf{s}}=Q_{p o} E X\left(1+P / K_{l}\right)^{-1},
$$

where $Q_{p o}$ is the specific enzyme activity $(0.6 \mathrm{~g} \mathrm{CH} / \mathrm{g}$ protein $\cdot \mathrm{h})$. The accumulation of enzyme was also taken as a balance between the rates of synthesis and degradation, with cycloheximide acting to repress enzyme synthesis:

$$
\frac{d E}{d t}=K_{\mathrm{S}}\left(1+P / K_{\mathrm{R}}\right)^{-1}-K_{\mathrm{de}} E,
$$

where $K_{\mathrm{S}}$ is the rate of enzyme synthesis $\left(6.0 \times 10^{-5} \mathrm{~g}\right.$ enzyme/g cells $\left.\cdot \mathrm{h}\right), K_{\mathrm{R}}$ is the feedback repression constant $(0.1 \mathrm{~g} \mathrm{CH} / \mathrm{L})$, and $K_{\mathrm{de}}$ is the degradation rate of the enzyme $\left(8.0 \times 10^{-3} \mathrm{~h}^{-1}\right)$. Evaluation of the parameters in equations 7 and 8 , though, was problematic because there were no experimental data characterizing the biosynthetic enzymes for cycloheximide synthesis. The values given above were found essentially by trial and error to give the closest agreement with the experimental cycloheximide profile.

In testing the enzyme model, the effect of induction of enzyme synthesis together with feedback inhibition (no feedback repression) was tested first. This was accomplished by setting the feedback repression constant, $K_{\mathrm{R}}$ in equation 8 , to a large value. As shown in FIGURE 4, this model yielded a significant improvement in the agreement with the typical batch data. However, in the simulated fed-batch fermentation, the

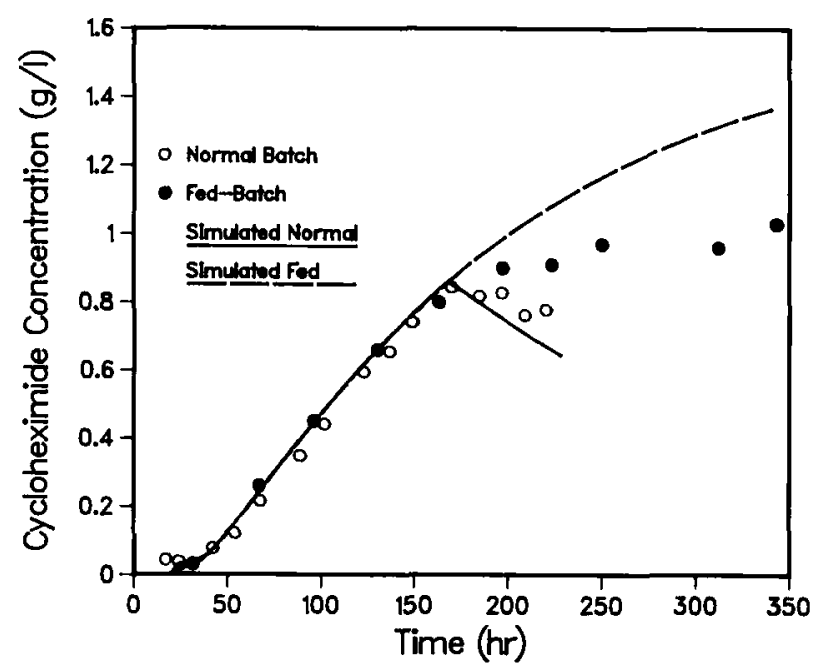

FIGURE 4. Results obtained using the induction-plus-inhibition model. The simulation fits the normal batch data very well, but it does not agree with complete feedback regulation data. 


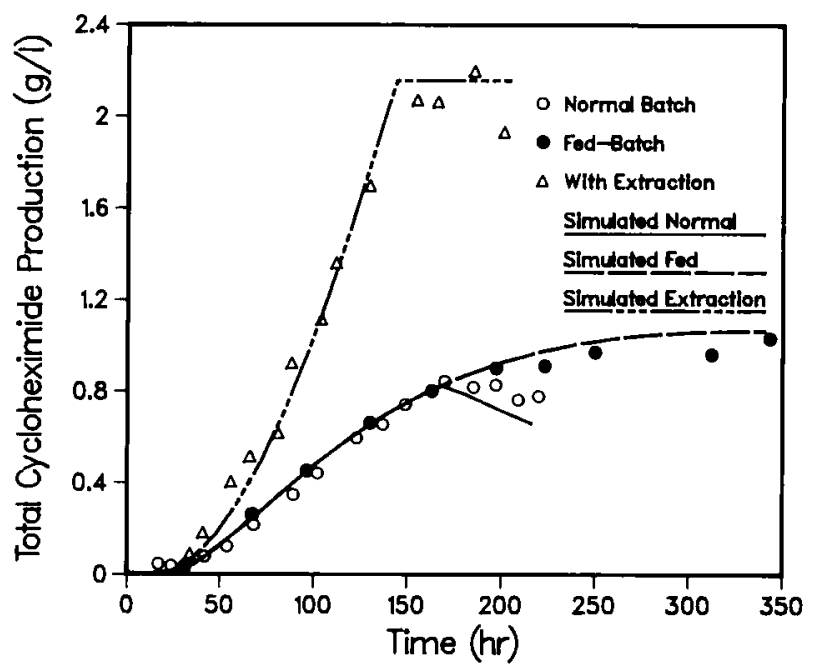

FIGURE 5. Simulated effect of induction plus feedback repression. This model fit all observed fermentation data very well.

effect of inhibition alone was not strong enough to explain the experimentally observed cessation of cycloheximide accumulation.

By setting the inhibition constant, $K_{l}$, to a large value, the effect of induction and feedback repression could be examined. In this simulation, cycloheximide accumulation affects the amount of synthesizing enzymes present, rather than the activity of the enzymes. As shown in FIGURE 5, the induction-plus-repression model does a good job of describing the cycloheximide profiles for all three types of fermentations. In addition, the simulated profile for the rate of antibiotic synthesis (FIGURE 3 ) shows qualitative agreement with the experimental profile. When the effects of both inhibition and repression were combined with induction (data not shown), there was little improvement in the fit over that achieved using only repression plus induction. We thus see that feedback repression appears to best account for the observed experimental data in this system.

\section{EXPERIMENTAL: TWO-DIMENSIONAL ELECTROPHORESIS STUDIES}

In the above computer model, the mode of feedback regulation was hypothesized to be repression of an unknown, rate-limiting enzyme by cycloheximide. Experimental verification of this model would involve tracking the enzyme concentration as a function of time in a normal batch fermentation and then observing the effect of various fermentation conditions on this enzyme profile. However, devising a specific experimental test for the presence of this enzyme is problematic because the biosynthetic pathway for cycloheximide has yet to be elucidated. An alternative to assaying for a specific enzyme activity is to examine how the overall protein profile of the 
microorganism changes over the course of the normal fermentation and under various conditions. Two-Dimensional Polyacrylamide Gel Electrophoresis (2-D PAGE) has been variously used to study the effects of heat shock on $E$. coli, to study sporulation in Saccharomyces cerevisiae, ${ }^{8}$ and to examine changes in specific protein levels due to drug treatment in rat fat cells.' In addition, quantitative information about specific protein levels has been gathered using a videocamera and microcomputer to perform optical densitometry on the gels. ${ }^{10-12}$ Thus, 2-D PAGE appears to be an ideal method for studying the effects of various fermentation conditions on protein levels in Streptomyces griseus.

\section{Materials and Methods}

\section{Fermentation}

In this work, Streptomyces griseus, strain UC-2132, donated by UpJohn Corporation (Kalamazoo, Michigan), was used throughout. Seed cultures were inoculated with a $1-\mathrm{mL}$ aliquot of frozen culture ( $50 \%$ glycerol, $50 \%$ culture) into a 2 - $\mathrm{L}$ shake flask containing $500 \mathrm{~mL}$ of seed medium. The seed medium contained glucose, $10 \mathrm{~g}$; beef extract, $5 \mathrm{~g}$; peptone, $5 \mathrm{~g} ; \mathrm{NaCl}, 5 \mathrm{~g}$; and tap water (to $1 \mathrm{~L}$ ). The fermentations were grown in $500-\mathrm{mL}$ shake flasks with $50-\mathrm{mL}$ fermentation volumes. The production medium contained cerelose, $66 \mathrm{~g}$; defatted soy flour, $15 \mathrm{~g}$; yeast extract, $2.5 \mathrm{~g}$; $\left(\mathrm{NH}_{4}\right)_{2} \mathrm{SO}_{4}, 5 \mathrm{~g} ; \mathrm{NaCl}, 4 \mathrm{~g} ; \mathrm{KH}_{2} \mathrm{PO}_{4}, 0.2 \mathrm{~g} ; \mathrm{CaCO}_{3}, 8 \mathrm{~g}$ (for $\mathrm{pH}$ control); and tap water (to $1 \mathrm{~L}$ ). Cycloheximide was assayed using the colorimetric method of Takeshita et al. ${ }^{13}$ Glucose was determined by the method of Somogyi, ${ }^{14}$ and total cell protein was determined by the Bradford assay. ${ }^{15}$

\section{Two-Dimensional Polyacrylamide Gel Electrophoresis}

2-D PAGE was performed essentially using the protocol of O'Farrell, ${ }^{16}$ with the following two modifications.

Sample Preparation. For each data point, a shake-flask fermentation was sacrificed. The cells were removed from the whole broth by centrifugation and the supernatant was saved for later assay. Following resuspension in an equal volume of sonication buffer, the cells were subjected to sonication for $3 \mathrm{~min}$. The 3 -min total sonication time was divided into 30-s intervals, which were interspersed with $30 \mathrm{~s}$ in an ice bath. Oversonification of the sample led to protein degradation and this resulted in "smeared" gels. The cell lysate was then centrifuged extensively to remove all insolubles. Following assay of the total protein, $4 \mathrm{mg}$ of total cell protein was precipitated with 5\% TCA. The precipitated protein was resuspended in $100 \mu \mathrm{L}$ sonication buffer, which was followed by addition of $100 \mathrm{mg}$ urea and $200 \mu \mathrm{L}$ lysis buffer to give a final volume of approximately $400 \mu \mathrm{L}$. This solution was recentrifuged to remove undissolved protein and urea before loading roughly $40-50 \mu \mathrm{L}(\sim 400 \mu \mathrm{g}$ protein) onto the first dimension gel.

Electrophoresis. Equilibrium isoelectric focusing was carried out for $14 \mathrm{~h}$ at a constant voltage of $400 \mathrm{~V}$, followed by $1 \mathrm{~h}$ at $1000 \mathrm{~V}$. The second-dimension slab gels 
were poured using AcrylAide crosslinker instead of bis-acrylamide and GelBond PAG film (FMC BioProducts, Rockland, Maine) to promote easy drying of the gels. Following silver staining and impregnation with $5 \%$ glycerol, the gels were allowed to air-dry. Silver staining of the gels was carried out using the protocol of Heukeshoven and Dernick. ${ }^{17}$ To compensate for the reduced diffusional area from the PAG film, each of the soaking times was doubled.

\section{Quantitation of Protein Spots}

Quantitation of the various individual proteins was carried out by using a videocamera-based image analysis system to measure the integrated optical density of a protein spot. This system consists of a solid-state videocamera (Javelin, Torrance, California; Model 2064IR) interfaced with a digitizing board for the IBM PC-AT (Imaging Technology, Woburn, Massachusetts; Model PFG-8-3) and a color video

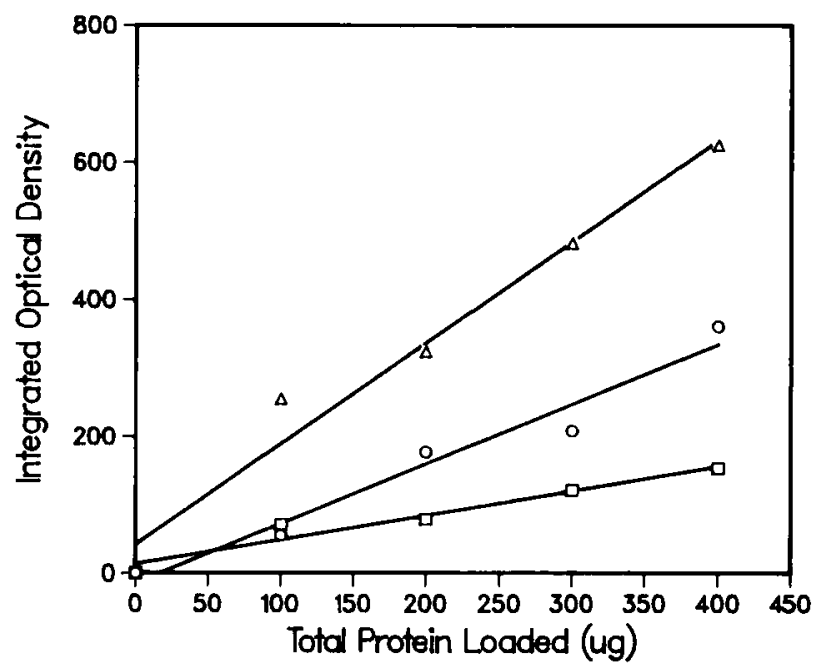

FIGURE 6. Plot of IOD versus amount of protein loaded onto the gels. The figure shows the linear relationship between the integrated optical density and the amount of protein in a spot.

monitor (Electrohome, Ontario, California; Model ECM 1311). The raw intensity data was transformed into integrated OD (IOD) values using ImageMeasure image analysis software (Microscience, Federal Way, Washington).

To account for variations in the incident light levels for different gels and to adequately resolve closely packed or overlapping protein spots, it was necessary to systematically define those pixels to be included into the integrated OD measurement. In this work, a threshold intensity was used to define the pertinent region of a protein spot. This threshold was set at the average between the local incident and the minimum intensities for a particular spot. Pixels with intensity lower than the threshold were included in the measurement, while pixels with higher intensity were discarded. 


\section{Results}

In a typical two-dimensional electrophoretogram, hundreds of proteins can be clearly resolved. However, a crucial question to be answered is whether or not individual protein concentrations can in fact be reproducibly measured. In order to check the relationship between the measured integrated optical density and the protein concentration, different amounts of a single protein sample were loaded onto each of six gels and the IOD of three individual proteins was measured. As shown in FIGURE 6, there clearly is a linear relationship between the IOD and the amount of protein in a spot for each of the three proteins assayed. It is also interesting to note that for each protein, the line of IOD versus protein loaded passes through the origin (within experimental error). This result suggests that it would be possible to assess the relative amount of a particular protein without actually knowing the absolute amount of protein loaded. If several proteins could be identified whose concentrations relative to one another remain constant over the course of the fermentation, then changes in the level of a particular protein of interest could be quantified by comparing its IOD to that of the constant proteins. In this manner, it would be possible to compare various protein samples without being concerned with experimental variations between different electrophoresis runs.

In fact, comparison of a number of protein samples taken at various fermentation times revealed a group of proteins whose concentrations relative to one another remained constant to within $\pm 26 \%$. At the same time, several other proteins showed significant variation with fermentation time. FIGURE 7 shows a comparison of

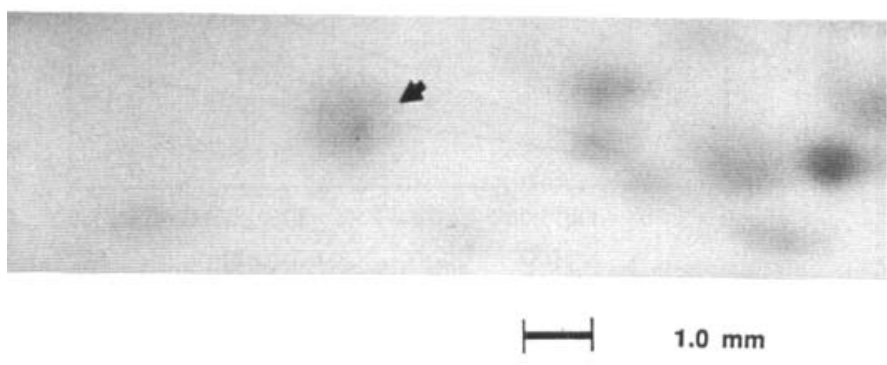

B

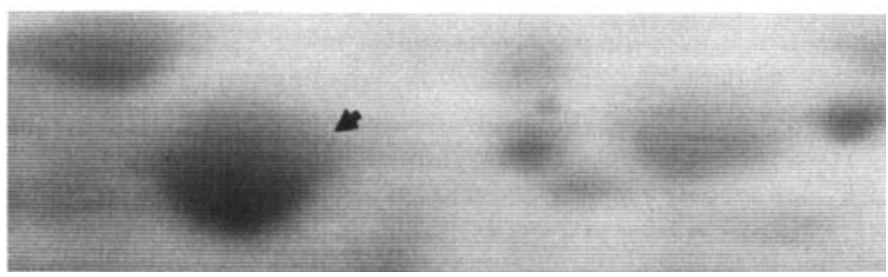

FIGURE 7. Comparison of corresponding gel regions for protein samples at (A) $t=2$ days and (B) $t=4$ days. 


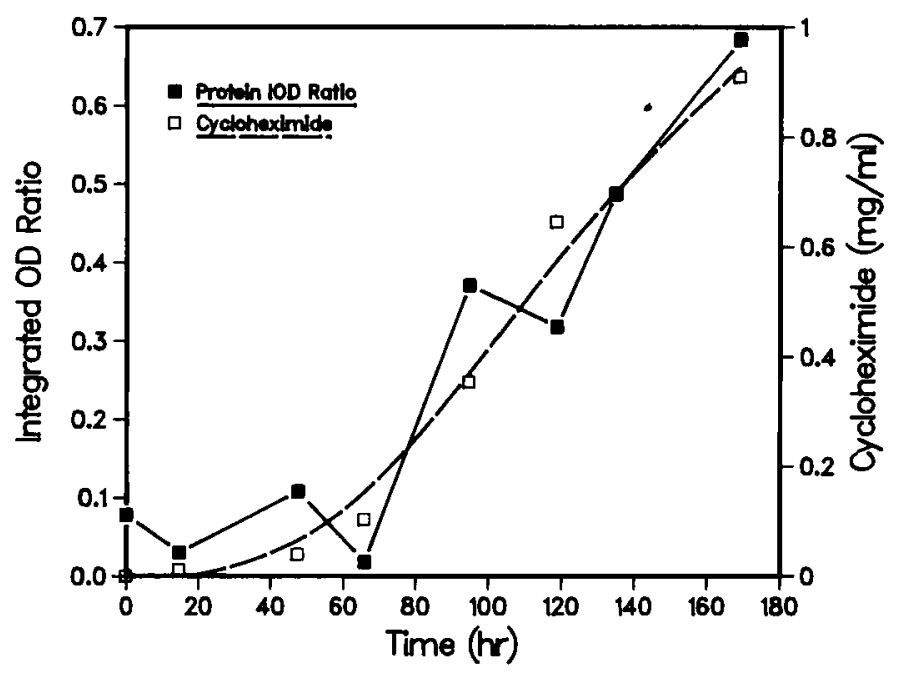

FIGURE 8. Change in the relative level of a particular protein with fermentation time.

corresponding regions on gels for protein samples taken at fermentation times of two days (FIGURE 7A) and four days (FIGURE 7B). It is easily observed that while the proteins shown on the right-hand side of the region are present at similar levels over the two-day interval, the proteins on the left-hand side of FIGURE 7B ( $t=$ four days) are either not present at two days or have dramatically increased in concentration over the two-day interval.

When the concentration of a particular protein (marked with an arrow in FIGURE 7) was followed with fermentation time, its relative level was found to change as shown in FIGURE 8. The rough correlation between the relative level of this protein and the extracellular cycloheximide concentration is clearly apparent, although the relationship (if any) between the two is unclear from these preliminary studies.

\section{CONCLUSIONS}

This work illustrates several interesting points about the cycloheximide fermentation. First, in modeling the production of cycloheximide, there are fundamental problems with using a constant amount of synthesizing enzyme plus feedback inhibition alone to describe initiation and feedback regulation of cycloheximide synthesis. However, models where enzyme synthesis is induced give a substantial improvement in the agreement between the simulated cycloheximide profile and the experimental data. In addition, models simulating a degree of regulation at the genetic level-that is, feedback repression-fit experimental data in all cases as well as or better than models where feedback inhibition is the only mechanism of regulation. These results indicate a possible relationship between protein synthesis and cycloheximide production.

Two-Dimensional Polyacrylamide Gel Electrophoresis was used to examine this 
relationship and several proteins were identified whose relative levels underwent interesting changes over the course of the fermentation. Future work will focus on relating these changes to production of cycloheximide. Using the 2-D PAGE techniques outlined here, it will be possible to examine the effects of various fermentation conditions, such as fermentation with on-line product extraction, on the protein profile of Streptomyces griseus.

\section{REFERENCES}

1. KomineK, L. A. 1975. Antimicrob. Agents Chemother. 7(6): 861 .

2. Wang, H. Y. 1983. Ann. N.Y. Acad. Sci. 431: 313.

3. BAILEY, J. E. \& D. F. OlLIS. 1977. Biochemical Engineering Fundamentals (1st edition), p. 407. McGraw-Hill. New York.

4. RoBINSON, F. E. \& H. Y. WANG. 1981. Unpublished experimental results. University of Michigan.

5. Payne, G. F. 1984. Ph.D. dissertation. University of Michigan.

6. Votruba, J. \& V. Behal. 1984. Appl. Microbiol. Biotechnol. 19: 153-156.

7. Herendeen, S. L., R. A. Van Bogelen \& F. C. Neidhardt. 1979. J. Bacteriol. 139(1): $185-194$.

8. Lorincz, A. T., M. J. Miller, N-H. Xuong \& E. P. Geiduschek. 1982. Mol. Cell. Biol. 2(12): 1532-1549.

9. Schoenle, E. J., I. K. Adams \& D. W. Sammons. 1984. J. Biol. Chem. 259: 1211212116.

10. Mariash, C. N., S. Seelig \& H. H. Oppenheimer. 1982. Anal. Biochem. 121: 388-394.

11. Tracy, R. P. \& D. S. Young. 1984. Clin. Chem. 30: 462-465.

12. HARMON, J. D. \& W. G. OWEN. 1986. Biotechniques 4(3): 264-271.

13. Takeshita, M., H. Takahashi \& T. Okuda. 1962. Chem. Pharm. Bull. 10: 304-308.

14. SOMOGYI, M. 1945. J. Biol. Chem. 160: 69-73.

15. BRADFORD, M. 1976. Anal. Biochem. 72: 248-254.

16. O'FARrell, P. H. 1975. J. Biol. Chem. 250(10): 4007-4021.

17. Heukeshoven, J. \& R. Dernick. 1985. Electrophoresis 6: 103-112. 